\title{
Cardiovascular magnetic resonance clarifies arrhythmogenicity in asymptomatic young athletes with ventricular arrhythmias undergoing pre-participation evaluation
}

\author{
GEORGE MARKOUSIS-MAVROGENIS ${ }^{1}$, AIKATERINI GIANNAKOPOULOU ${ }^{2}$, NIKOLAOS ANDREOU ${ }^{2}$, \\ GEORGE PAPADOPOULOS $^{2}$, VASILIKI VARTELA ${ }^{1}$, GENOVEFA KOLOVOU ${ }^{1}$, \\ FLORA BACOPOULOU $^{3}$, KONSTANTINOS TSAROUHAS ${ }^{3,4}$, CHRISTINA KANAKA-GANTENBEIN ${ }^{3}$, \\ DEMETRIOS A. SPANDIDOS ${ }^{5}$ and SOPHIE I. MAVROGENI ${ }^{1,3}$
}

\begin{abstract}
${ }^{1}$ Onassis Cardiac Surgery Center, Athens $17674 ;{ }^{2}$ Department of Cardiology, Aghia Sophia Children's Hospital, Athens 11527; ${ }^{3}$ Exercise Physiology and Sports Medicine Clinic, Center for Adolescent Medicine and UNESCO Chair on Adolescent Health Care, First Department of Pediatrics, Medical School, National and Kapodistrian University of Athens, Aghia Sophia Children's Hospital, Athens 11527; ${ }^{4}$ Department of Cardiology, University Hospital of Larissa, Larissa 41334; ${ }^{5}$ Laboratory of Clinical Virology, School of Medicine, University of Crete, Heraklion 71003, Greece
\end{abstract}

Received April 2, 2020; Accepted April 29, 2020

DOI: $10.3892 / \mathrm{etm} .2020 .8693$

\begin{abstract}
Pre-participation sports examination (PPE) is a frequent reason for consultation. However, the exact role of cardiovascular magnetic resonance (CMR) in PPE remains undefined. The additive value of CMR in adolescent athletes with ventricular rhythm disturbances (VRDs) was investigated. We prospectively recruited and evaluated with CMR 50 consecutive, asymptomatic young athletes referred to our tertiary center after identification of VRDs on electrocardiogram (ECG) with otherwise normal standard PPE and echocardiography, and 20 age- and sex-matched healthy
\end{abstract}

Correspondence to: Professor Sophie I. Mavrogeni, Onassis Cardiac Surgery Center, 107-109 Syngrou Avenue, Athens 17674, Greece

E-mail: sophie.mavrogeni@gmail.com

Abbreviations: PPE, pre-participation sports examination; CMR, cardiovascular magnetic resonance; VRD, ventricular rhythm disturbance; ECG, electrocardiogram; VT, ventricular tachycardia; LGE, late gadolinium enhancement; EGE, early gadolinium enhancement; ECV, extracellular volume fraction; SCD, sudden cardiac death; HCM, hypertrophic cardiomyopathy; PVC, premature ventricular contractions; MOLLI, modified Look-Locker inversion; LV, left ventricle; RV, right ventricle; ESV, end-systolic volume; EDV, end-diastolic volume; EF, ejection fraction; ARVC arrhythmogenic right ventricular cardiomyopathy; DMD Duchenne muscular dystrophy; BMD, Becker muscular dystrophy; MCP, minmax concave penalty; mFDR, marginal false discovery rate; LASSO, least absolute shrinkage and selection operator; BSA, body surface area

Key words: ventricular arrhythmia, sudden cardiac death, cardiovascular magnetic resonance, athlete; pre-participation sports evaluation, penalized regression volunteer athletes who underwent the same evaluations. The primary outcome was case-control status and the secondary outcome was the discrimination between athletes with VRDs with and without non-sustained ventricular tachycardia (VT). CMR identified arrhythmogenic substrates in all athletes with VRDs. The predominant condition was myocarditis and arrhythmogenic right ventricular cardiomyopathy in patients with and without VT, respectively. Based on penalized regression analysis, late gadolinium enhancement (LGE), early gadolinium enhancement (EGE), extracellular volume fraction (ECV), and T2-mapping, best distinguished between case-control status. The aforementioned indices predicted case-control status independent of age and sex: EGE [Odds ratio (95\% confidence interval): 6.89 (2.19-21.62) per 0.5-unit, $\mathrm{P}<0.001], \mathrm{LGE}$ (perfect prediction), ECV [1.66 (1.25-2.22), $\mathrm{P}<0.001]$ and $\mathrm{T} 2$ mapping [1.40 (1.13-1.72), $\mathrm{P}=0.002]$, among other independent CMR-derived predictors. Only indexed ventricular volumes independently discriminated between VRD patients with and without VT. In this study, asymptomatic young athletes with VRDs and normal PPE/echocardiography were optimally discriminated from healthy control athletes by CMR-derived indices, and CMR allowed for the identification of arrhythmogenic substrates in all cases.

\section{Introduction}

The pre-participation sports examination (PPE) is a frequent reason for consultation for children and adolescents wishing to engage in sports at the amateur or professional level (1). The ultimate goal of PPE is to ensure the safe participation of athletes in sporting activities, most importantly by precluding the existence of conditions predisposing to sudden cardiac death (SCD) during exercise (2). Traditionally this involves the taking of a focused patient history and the performance of a physical examination of the cardiovascular and musculoskeletal 
systems (1,3). More recently, electrocardiogram (ECG) and echocardiographic evaluation have also been gaining ground in the context of PPE $(4,5)$. However, PPE has not been proven to significantly reduce the burden of morbidity/mortality associated with athletic activities (1). This may potentially be attributed to the inability of currently employed PPE clinical algorithms and/or diagnostic modalities to adequately detect patients at high risk for SCD (6).

Supplementary cardiovascular imaging may offer incremental diagnostic value within existing PPE practices, and this is currently primarily represented by echocardiography. Although echocardiography is a widely available, low-cost imaging modality that does not require the use of ionizing radiation, it is limited by operator- and window-dependency, which can limit reproducibility and diagnostic accuracy, respectively (7). Importantly, echocardiography is unable to characterize myocardial tissues with regard to the presence of fibrosis or edema and may even miss structural alterations including apical cardiomyopathy (7-9).

Cardiovascular magnetic resonance (CMR) is the only imaging modality that can compensate for these limitations of echocardiography (10). Namely, CMR allows for the identification of arrhythmogenic substrates in patients with normal echocardiographic evaluation (11). This is of particular importance in young individuals, as cardiac lesions in this population may initially present directly with ventricular rhythm disturbances (VRDs) and subsequent SCD without prior warning or clinical indications, notably even if echocardiographic evaluation is normal (3). Additionally, untreated young patients with ventricular tachycardia (VT) have a worse prognosis (12) and thus the identification of clinically silent arrhythmogenic substrates is of paramount importance for the optimization of PPE.

CMR has been studied in adult patients with VRDs and congenital heart disease $(13,14)$ and has been shown to identify children with hypertrophic cardiomyopathy (HCM) at risk of VT (15). However, little is known regarding the clinical significance of CMR in the context of PPE. It was hypothesized that in a cohort of young athletes with ECG-demonstrated VRDs and a normal standard PPE and echocardiographic examination, CMR could match the underlying VRDs to arrhythmogenic substrates in the myocardium. Our aim was to evaluate a population of young athletes with VRDs as well as normal echocardiograms and routine PPE using CMR, in order to identify potential arrhythmogenic substrates and to compare their CMR findings with those of a healthy young control athlete population without VRDs.

\section{Patients and methods}

Participants. Prospectively 50 consecutive, asymptomatic, young athletes (henceforth referred to as patients), aged up to 18 years, referred to our tertiary center were recruited (Aghia Sophia Children's Hospital, Athens, Greece) from primary care providers of PPE, after identification of VRDs on ECG with otherwise normal standard PPE and echocardiography. They were compared with 20 age- and sex-matched healthy control athletes, who underwent the same evaluations and had no objectifiable abnormalities or symptoms. Healthy control athletes were recruited from a pool of voluntary participants and case-control status was determined a priori based on the presence of any ECG abnormalities, since all study participants were asymptomatic and with normal echocardiograms. CMR findings were not taken into account for the definition of case-control status. VRDs included premature ventricular contractions (PVC) such as bigeminy, trigeminy, couples or triplets and non-sustained VT. Our institution is the national reference center for PPE evaluation, including further evaluation of athletes with abnormal PPE screening. All participants taking part in this study were re-screened at our institution for symptoms related to the cardiovascular system using a standardized structured approach (4). This included a detailed individual and family history, clinical evaluation, ECG and echocardiogram, all reviewed by the same physician. The entire cohort was subsequently evaluated using a comprehensive CMR protocol. The study was approved by the medical ethics committee of the Aghia Sophia Children's Hospital (protocol no. 27499/29-11-17) and written informed consent was obtained from the parents or legal guardians of the participants.

CMR evaluation. CMR examinations were performed using a 1.5-T scanner (Ingenia, Philips Medical Systems). The CMR protocol included standard steady-state free-precession cine CMR, black-blood T2-weighted short tau inversion recovery images, T1-weighted spin-echo early gadolinium enhancement (EGE) images, and phase-sensitive inversion recovery late gadolinium enhancement (LGE) images as described previously (16). A dose of $0.1 \mathrm{mmol} / \mathrm{kg}$ gadobenatedimeglumine contrast-medium was injected for EGE images and another $0.1 \mathrm{mmol} / \mathrm{kg}$ for LGE images (16).

T1-mapping was performed using a modified Look-Locker inversion recovery (MOLLI) sequence with a 3(3)5 scheme on 3 representative short-axis positions immediately before and 15 min after contrast-medium administration. T2-mapping was performed on 3 representative LV short axis slices using a black-blood prepared, navigator-gated, free-breathing hybrid gradient (echo planar imaging) and spin-echo multiecho sequences (11).

CMR data analysis. Short axis steady-state free-precession cine CMR was used to evaluate biventricular function [left and right ventricular (LV/RV) end-systolic/-diastolic volumes (ESV/EDV) and ejection fractions (EF)] following standard practice (17). Global myocardial inflammation was assessed using T2-weighted images by calculating the T2 signal intensity ratio of myocardium to skeletal muscle (16). Global relative enhancement was calculated by measuring myocardial signal intensity on pre- and post-contrast T1-weighted spin-echo images relative to skeletal muscle (16). The presence and pattern of non-ischemic LGE lesions were qualitatively assessed by consensus agreement of 2 experienced observers and expressed as a percentage of LV mass (\%LGE). Native and post-contrast T1-mapping, the extracellular volume fraction (ECV) and T2-mapping values were generated using dedicated plugins written for the OsiriX software as described previously (18). Global native/post-contrast myocardial T1, ECV, and T2 values were calculated as the mean value of 3 short-axis slices.

Validation of $T 1$ and T2 measurements. The accuracy of the T1- and T2-mapping methods was evaluated with a relaxometry 
study using a Eurospin Gel-Phantom (TO5, Diagnostic Sonar LTD, Livingston, Scotland): the comparison of T1 values obtained by the MOLLI 3(3)5 and a reference scan has been previously reported (19). T2 values obtained using the black-blood-prepared multiecho hybrid gradient and spin-echo sequence were compared with a spin-echo reference sequence with 16 echoes, 8 -msec echo spacing, and 10-sec time to repetition. Furthermore, myocardial T2 values were measured in 16 myocardial segments in an additional control group to assess reproducibility and regional variations of estimated myocardial T2 signals (19). This control group consisted of 20 healthy, asymptomatic subjects (median age, 15 years [IQR, 12-18 years]) without cardiovascular disease. Inter-scan reproducibility was assessed for myocardial $\mathrm{T} 1$ and $\mathrm{T} 2$ measurements by performing 10 repeated scans with identical imaging parameters. An inter-observer agreement of 0.85 was observed between 2 blinded observers in all subjects.

Locally determined cut-off values for CMR variables. Locally determined cut-off points for tissue characterization indices were $\mathrm{LGE}>0 \%$, EGE $>4$, T2 signal ratio $>1.9$, native T1-mapping $>1,050 \mathrm{msec}$, post-contrast T1-mapping $<350 \mathrm{msec}$, T2-mapping $>55 \mathrm{msec}$ and ECV $>28 \%$.

Diagnostic criteria. Where applicable, the diagnosis of acute/chronic infectious/non-infectious myocarditis was based on the 2009 Lake Louise criteria (16), that of arrhythmogenic right ventricular cardiomyopathy (ARVC) on the Task Force Criteria for pediatric ARVC (20), that of non-compaction cardiomyopathy on published criteria for non-compaction cardiomyopathy in children (21) and that of Duchenne/Becker muscular dystrophy (DMD/BMD) patient/carrier status on the presence of subepicardial LGE in the lateral LV wall (22).

Statistical analysis. Statistical analyses were carried out using Stata v.15SE and R v.3.6.0. Normally distributed variables are presented as mean (standard deviation), continuous not-normally distributed variables are presented as median (interquartile range) and binary/categorical variables are presented as number (percentage). The primary outcome was patient vs. control status and the secondary outcome was patients with VT vs. patients without VT. Descriptive statistics were determined and evaluated for statistical significance between each of the groups per outcome using the independent-sample t-test, Mann-Whitney U test and Chi-square test for normally distributed, not-normally distributed continuous and binary/categorical variables, respectively. Similarly, when comparing more than two groups, a one-way analysis of variance, the Kruskal-Wallis test and the chi-square test were used, respectively.

Logistic regression analyses were used to apply multivariable corrections for age and sex to CMR predictors of the primary and secondary outcomes. The ncvreg package (23) was used for performing $\mathrm{min} / \mathrm{max}$ concave penalty (MCP) logistic regression analyses with k-fold cross-validation for both outcomes in order to inform variable selection for multivariable models. Indexed ventricular volumes, ventricular ejection fractions and tissue characterization indices, age and sex were included as potential features to be selected. The optimal value for the penalization term $\lambda$ was determined as the value that minimizes the cross-validation error rate. The reliability of selected features was evaluated using the built-in marginal false discovery rate (mFDR), which performs better than other inference methods for penalized regression analyses $(24,25)$. Signal-to-noise ratios are also presented. Model predictive capacities are reported as cross-validated $\mathrm{R}^{2}$ values. Penalized regression analyses often overcome the disadvantages of stepwise or best subset approaches for feature selection (26) and allow for the selection of important predictors by optimizing the variance-bias trade-off (27). This in turn increases the external validity of the identified predictors at the cost of more biased estimates. The employed type of penalization (MCP) has been shown to be less biased towards features with larger coefficients than other penalization methods such as least absolute shrinkage and selection operator (LASSO) $(23,26)$ and was thus preferred for statistical analyses presented in this manuscript.

\section{Results}

Baseline characteristics of the included patients and control athletes are presented in Table I. The median ages (IQR) of the former and latter were 13.5 (11.0-17.0) and 15 (12.0-18.0) years, respectively $(\mathrm{p}=0.37)$. Similarly, the proportion of females was $19(38 \%)$ and $10(50 \%)$, respectively $(\mathrm{P}=0.36)$. Regarding the previous medical history of the patient group, $1(2 \%)$ patient had known Hashimoto's thyroiditis and was under appropriate endocrinologic treatment. The remainder of the patient cohort $(n=49,98 \%)$ had no known past medical history. The totality of the patient cohort had no objectifiable cardiovascular symptoms. For each of the 50 asymptomatic patients with normal echocardiographic evaluation and documented VRDs, a clinical diagnosis was made based on CMR findings in combination with clinical and diagnostic information. Namely, 20 (40\%) were diagnosed with recent-onset myocarditis, 8 (16\%) with remitting past myocarditis (previous PPE with ECG was normal in these participants), 9 (18\%) with ARVC, 5 (10\%) with non-compaction cardiomyopathy, 2 (4\%) with female-carrier status of DMD, $3(6 \%)$ males with BMD, $1(2 \%)$ with dilated cardiomyopathy, 1 (2\%) with Hashimoto thyroiditis with cardiac involvement and 1 (2\%) with HCM. The aforementioned diagnoses were not known at the time of CMR evaluation.

Patients had significantly higher indexed diastolic ventricular volumes compared with control athletes and tissue characterization indices differed significantly between groups (Table I). This was especially the case for LGE which was identified in none of the controls, but in $40(80 \%)$ of the patients $(\mathrm{P}<0.0001)$. Native T1-mapping and ECV were similarly abnormal in a large majority of the patients compared with none of the controls [21 (42\%) and 24 (48\%), respectively, $\mathrm{P}<0.001$ for both]. No statistically significant differences were identified in LV/RVEF between the two groups. In contrast to the comparisons between patients and controls, few significant differences were identified between patients with and without VT (Table II). These were reflected by overall significantly smaller indexed biventricular volumes in the VT group without significant differences in LV/RVEF or tissue characterization indices. There was a trend for a smaller proportion of abnormal EGE values in the VT group compared with the non-VT group, 
Table I. Comparison of baseline characteristics between control athletes and asymptomatic athletes with VRD.

\begin{tabular}{|c|c|c|c|}
\hline Variable & Control athletes & Patients & P-value \\
\hline \multicolumn{4}{|l|}{ Demographics } \\
\hline Participant no. & 20 & 50 & N/A \\
\hline Female sex & $10(50 \%)$ & $19(38 \%)$ & 0.36 \\
\hline Age (years) & $15.0(12.0,18.0)$ & $13.5(11.0,17.0)$ & 0.37 \\
\hline \multicolumn{4}{|l|}{ Type of cardiac pathology } \\
\hline Recent-onset myocarditis & & $20(40 \%)$ & \\
\hline ARVC & & $9(18 \%)$ & \\
\hline Past myocarditis & & $8(16 \%)$ & \\
\hline $\mathrm{DMD} / \mathrm{BMD}$ & $\mathrm{N} / \mathrm{A}$ & $5(10 \%)$ & N/A \\
\hline Non-compaction cardiomyopathy & & $5(10 \%)$ & \\
\hline DCM & & $1(2 \%)$ & \\
\hline Hashimoto thyroiditis & & $1(2 \%)$ & \\
\hline LV hypertrophy & & $1(2 \%)$ & \\
\hline \multicolumn{4}{|l|}{ Types of rhythm disturbances } \\
\hline PVCs in couples & & $7(14 \%)$ & \\
\hline PVCs in triplets & & $9(18 \%)$ & \\
\hline Bigeminy & N/A & $9(18 \%)$ & N/A \\
\hline Trigeminy & & $4(8 \%)$ & \\
\hline VT & & $21(42 \%)$ & \\
\hline \multicolumn{4}{|l|}{ Ventricular volumes and function } \\
\hline LVEDV (ml) & $103.5(96.5,105.0)$ & $127.0(104.0,155.0)$ & $0.002^{\mathrm{a}}$ \\
\hline LVESV (ml) & $39.0(31.5,41.0)$ & $46.0(36.0,56.0)$ & $0.013^{\mathrm{a}}$ \\
\hline $\operatorname{LVEF}(\%)$ & $63.0(62.0,68.0)$ & $63.0(59.0,67.0)$ & 0.21 \\
\hline RVEDV (ml) & $100.0(92.5,113.5)$ & $126.0(97.0,144.0)$ & $0.011^{\mathrm{a}}$ \\
\hline RVESV (ml) & $41.5(33.0,49.0)$ & $49.0(34.0,63.0)$ & 0.17 \\
\hline RVEF (\%) & $59.5(54.0,63.5)$ & $62.0(59.0,64.0)$ & 0.12 \\
\hline \multicolumn{4}{|l|}{ Indexed ventricular volumes } \\
\hline LVEDV/BSA & $67.4(59.4,72.4)$ & $72.9(67.1,86.1)$ & $0.018^{\mathrm{a}}$ \\
\hline LVESV/BSA & $22.8(20.3,28.4)$ & $26.1(22.7,34.3)$ & 0.067 \\
\hline RVEDV/BSA & $63.2(52.9,72.6)$ & $73.4(61.3,88.0)$ & $0.043^{\mathrm{a}}$ \\
\hline RVESV/BSA & $25.4(22.2,30.9)$ & $28.5(21.1,36.2)$ & 0.45 \\
\hline \multicolumn{4}{|l|}{ Tissue characterization indices } \\
\hline EGE & $0.6(0.2,1.0)$ & $2.6(1.5,3.8)$ & $<0.001^{\mathrm{a}}$ \\
\hline LGE (\%) & $0.0(0.0,0.0)$ & $5.0(2.0,5.0)$ & $<0.001^{\mathrm{a}}$ \\
\hline T2 signal ratio & $1.4(1.2,2.0)$ & $2.0(1.8,2.4)$ & $<0.001^{\mathrm{a}}$ \\
\hline Native T1-mapping (msec) & $955.5(944.0,980.0)$ & $1,045.5(997.0,1,098.0)$ & $<0.001^{\mathrm{a}}$ \\
\hline Post-contrast T1-mapping (msec) & $467.0(455.0,478.5)$ & $445.0(414.0,481.0)$ & 0.064 \\
\hline $\mathrm{ECV}(\%)$ & $25.5(24.5,27.0)$ & $28.0(26.0,31.0)$ & $<0.001^{\mathrm{a}}$ \\
\hline T2-mapping (msec) & $47.0(43.0,49.5)$ & $50.0(48.0,53.0)$ & $<0.001^{\mathrm{a}}$ \\
\hline \multicolumn{4}{|c|}{ Locally-used normal values for tissue characterization indices } \\
\hline $\mathrm{EGE}>4$ & $0(0 \%)$ & $8(16 \%)$ & 0.057 \\
\hline $\mathrm{LGE}>0 \%$ & $0(0 \%)$ & $40(80 \%)$ & $<0.001^{\mathrm{a}}$ \\
\hline T2 signal ratio $>1.9$ & $6(30 \%)$ & $30(60 \%)$ & $0.023^{\mathrm{a}}$ \\
\hline Native T1-mapping $>1,050 \mathrm{msec}$ & $0(0 \%)$ & $21(42 \%)$ & $<0.001^{\mathrm{a}}$ \\
\hline Post-contrast T1-mapping $<350 \mathrm{msec}$ & $0(0 \%)$ & $4(8 \%)$ & 0.19 \\
\hline T2-mapping >55 msec & $0(0 \%)$ & $10(20 \%)$ & $0.031^{\mathrm{a}}$ \\
\hline $\mathrm{ECV}>28 \%$ & $0(0 \%)$ & $24(48 \%)$ & $<0.001^{\mathrm{a}}$ \\
\hline
\end{tabular}

${ }^{\mathrm{a}} \mathrm{P} \leq 0.05$. VRD, ventricular rhythm disturbance; ARVC, arrhythmogenic right ventricular cardiomyopathy; DMD/BMD, Duchenne/Becker muscular dystrophy; DCM, dilated cardiomyopathy; PVC, premature ventricular contraction; VT, non-sustained ventricular tachycardia; LV/RV, left/right ventricular; EDV/ESV, end-diastolic/-systolic volume; EF, ejection fraction; BSA, body surface area; EGE/LGE, early/late gadolinium enhancement; ECV, extracellular volume fraction. 
Table II. Comparison of baseline characteristics between VRD patients that did and did not experience VT.

\begin{tabular}{|c|c|c|c|}
\hline Variables & No VT & VT & $\mathrm{P}$-value \\
\hline \multicolumn{4}{|l|}{ Demographics } \\
\hline Participant no. & 29 & 21 & N/A \\
\hline Female sex & $13(45 \%)$ & $6(29 \%)$ & 0.24 \\
\hline Age (years) & $14.0(12.0,17.0)$ & $15.0(13.0,18.0)$ & 0.35 \\
\hline \multicolumn{4}{|l|}{ Type of cardiac pathology } \\
\hline Recent-onset myocarditis & $17(59 \%)$ & $3(14 \%)$ & \\
\hline ARVC & $0(0 \%)$ & $9(43 \%)$ & \\
\hline Past myocarditis & $5(17 \%)$ & $3(14 \%)$ & \\
\hline DMD/BMD & $2(7 \%)$ & $3(14 \%)$ & $0.001^{\mathrm{a}}$ \\
\hline Non-compaction cardiomyopathy & $4(14 \%)$ & $1(5 \%)$ & \\
\hline DCM & $0(0 \%)$ & $1(5 \%)$ & \\
\hline Hashimoto thyroiditis & $0(0 \%)$ & $1(5 \%)$ & \\
\hline LV hypertrophy & $1(3 \%)$ & $0(0 \%)$ & \\
\hline \multicolumn{4}{|l|}{ Ventricular volumes and function } \\
\hline LVEDV (ml) & $132.0(117.0,154.0)$ & $110.0(90.0,160.0)$ & 0.16 \\
\hline LVESV (ml) & $52.0(41.0,57.0)$ & $37.0(32.0,52.0)$ & 0.10 \\
\hline LVEF (\%) & $63.0(59.0,66.0)$ & $63.0(59.0,67.0)$ & 0.86 \\
\hline RVEDV (ml) & $134.0(106.0,156.0)$ & $115.0(78.0,127.0)$ & $0.035^{\mathrm{a}}$ \\
\hline RVESV (ml) & $51.0(43.0,68.0)$ & $43.0(29.0,54.0)$ & 0.072 \\
\hline RVEF (\%) & $62.0(60.0,63.0)$ & $62.0(56.0,64.0)$ & 0.92 \\
\hline \multicolumn{4}{|l|}{ Indexed ventricular volumes } \\
\hline LVEDV/BSA & $76.8(71.8,86.1)$ & $68.9(54.0,77.8)$ & $0.031^{\mathrm{a}}$ \\
\hline LVESV/BSA & $29.9(24.9,36.2)$ & $23.5(21.3,27.6)$ & $0.028^{\mathrm{a}}$ \\
\hline RVEDV/BSA & $81.2(69.2,92.3)$ & $69.3(49.6,76.1)$ & $0.019^{\mathrm{a}}$ \\
\hline RVESV/BSA & $32.8(27.3,36.6)$ & $25.5(19.4,33.5)$ & $0.019^{\mathrm{a}}$ \\
\hline \multicolumn{4}{|l|}{ Tissue characterization indices } \\
\hline EGE & $3.0(1.6,4.0)$ & $2.5(1.5,3.5)$ & 0.21 \\
\hline LGE $(\%)$ & $5.0(4.0,5.0)$ & $5.0(2.0,5.0)$ & 0.32 \\
\hline $\mathrm{T} 2$ signal ratio & $2.0(1.9,2.4)$ & $2.0(1.7,2.4)$ & 0.49 \\
\hline Native T1-mapping (msec) & $1,042.0(990.0,1,092.0)$ & $1,051.0(1,011.0,1,125.0)$ & 0.30 \\
\hline Post-contrast T1-mapping (msec) & $445.0(416.0,492.0)$ & $445.0(414.0,455.0)$ & 0.40 \\
\hline $\operatorname{ECV}(\%)$ & $28.0(26.0,31.0)$ & $28.0(26.0,31.0)$ & 0.81 \\
\hline T2-mapping (msec) & $50.0(48.0,53.0)$ & $50.0(48.0,52.0)$ & 0.55 \\
\hline \multicolumn{4}{|l|}{$\begin{array}{l}\text { Locally-used normal values for tissue } \\
\text { characterization indices }\end{array}$} \\
\hline $\mathrm{EGE}>4$ & $7(24 \%)$ & $1(5 \%)$ & 0.065 \\
\hline $\mathrm{LGE}>0 \%$ & $24(83 \%)$ & $16(76 \%)$ & 0.57 \\
\hline $\mathrm{T} 2$ signal ratio $>1.9$ & $18(62 \%)$ & $12(57 \%)$ & 0.73 \\
\hline Native T1-mapping $>1,050 \mathrm{msec}$ & $10(34 \%)$ & $11(52 \%)$ & 0.21 \\
\hline Post-contrast T1-mapping <350 msec & $2(7 \%)$ & $2(10 \%)$ & 0.74 \\
\hline T2-mapping $>55$ msec & $7(24 \%)$ & $3(14 \%)$ & 0.39 \\
\hline $\mathrm{ECV}>28 \%$ & $14(48 \%)$ & $10(48 \%)$ & 0.96 \\
\hline
\end{tabular}

${ }^{\mathrm{a}} \mathrm{P} \leq 0.05$. VT, non-sustained ventricular tachycardia; ARVC, arrhythmogenic right ventricular cardiomyopathy; DMD/BMD, Duchenne/Becker muscular dystrophy; DCM, dilated cardiomyopathy; LV/RV, left/right ventricular; EDV/ESV, end-diastolic/-systolic volume; EF, ejection fraction; BSA, body surface area; EGE/LGE, early/late gadolinium enhancement; ECV, extracellular volume fraction.

but this did not reach statistical significance [1 (5\%) vs. 7 (24\%), $\mathrm{P}=0.065]$. The predominant cardiac condition in the non-VT group was recent-onset myocarditis [17 (59\%)] while that of the VT group was ARVC [9 (43\%)] $(\mathrm{P}=0.001)$.
Results of univariable and multivariable logistic regression analyses are presented for both the primary and secondary outcomes in Table III. When adjusted for age and sex, indexed LVEDV and RVEF were significant positive predictors of the 


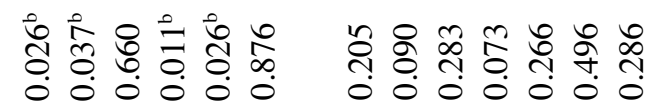

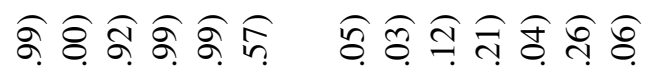

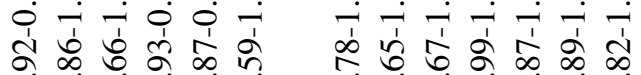

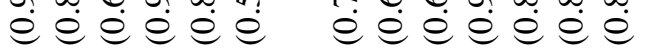

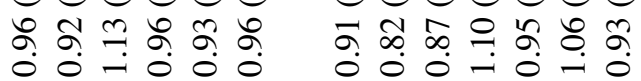

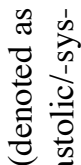

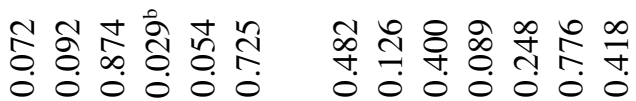

蒫

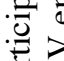

常证

学完

苟

幽

क

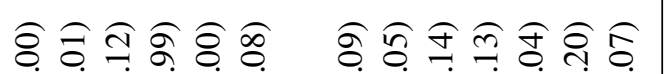

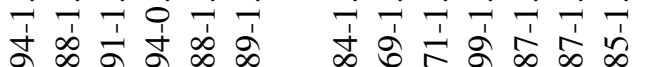

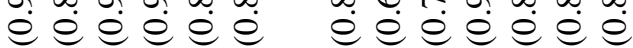

ڤ̆

궁

0 过

里

$>$

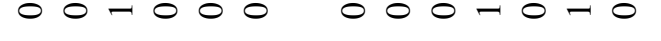

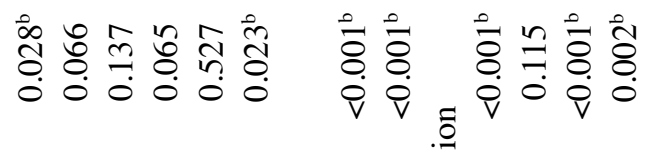

要

造造

政

on

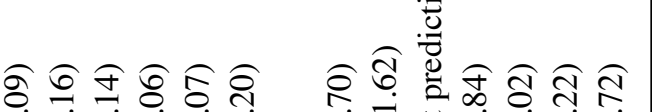

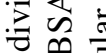

i.

조.

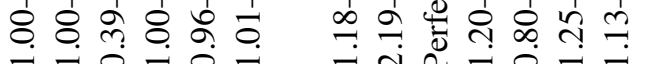

$\doteq \doteq \dot{\ominus} \doteq \dot{\vec{e}}$

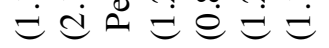

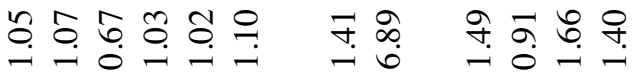

$8 \pm$

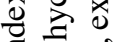

त证

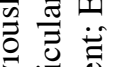

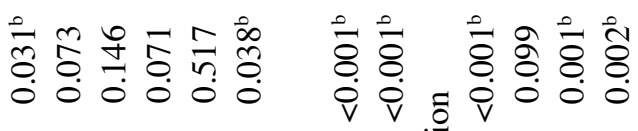

这泀

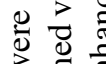

的

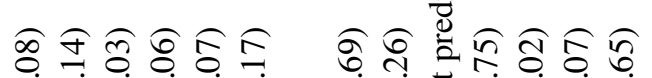

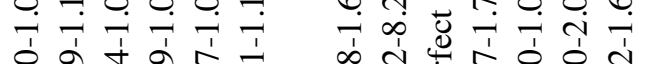

要春

융

츨

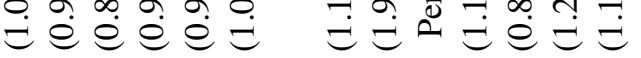

ఫิ

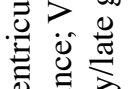

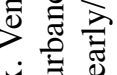

啳

를

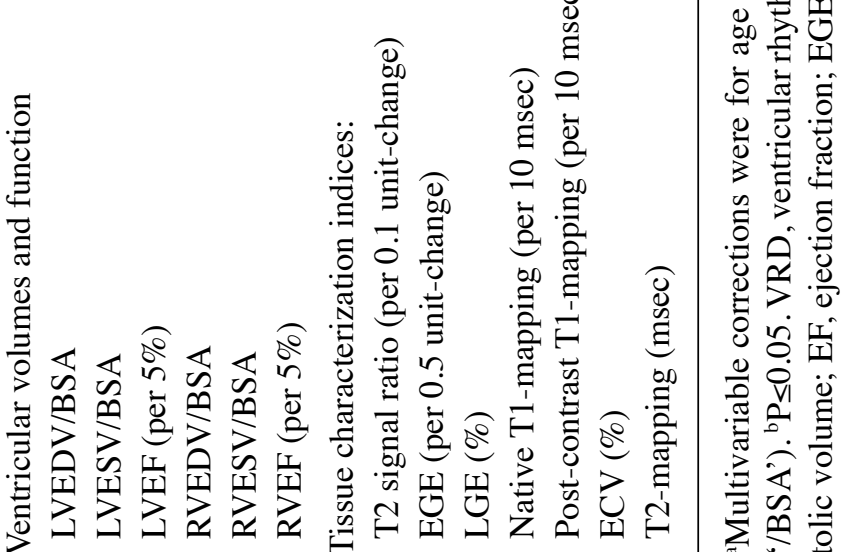

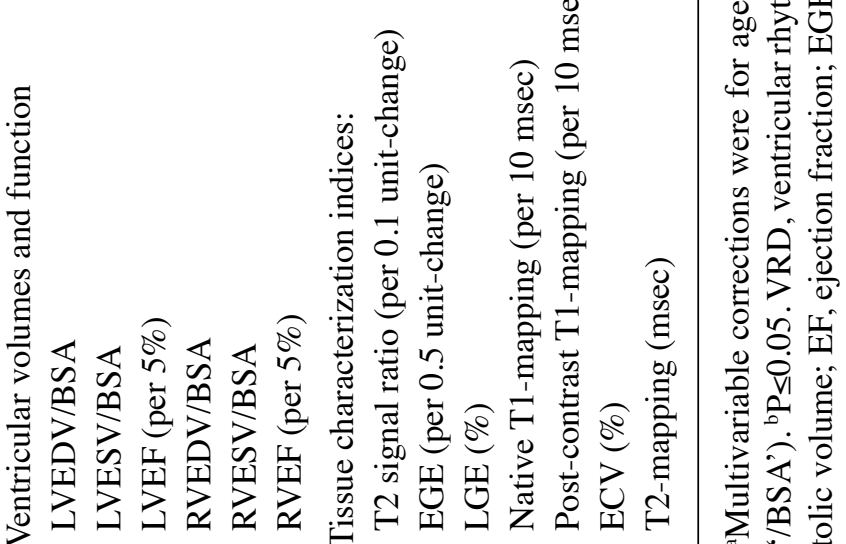


Table IV. Results of MCP-logistic regression analysis for differentiating VRD patients from healthy controls.

\begin{tabular}{|c|c|c|c|c|c|c|c|}
\hline Variables & Estimate & z-value & mFDR & $\begin{array}{l}\text { Average } \\
\text { mFDR }\end{array}$ & $\begin{array}{c}\text { Cross-validated } \\
\mathrm{R}^{2}\end{array}$ & $\begin{array}{c}\text { Signal-to-noise } \\
\text { ratio }\end{array}$ & $\begin{array}{l}\text { Prediction } \\
\text { error }\end{array}$ \\
\hline LGE $(\%)$ & 5.946 & 24.34 & 0.0001 & 0.266 & 0.63 & 1.68 & 0.029 \\
\hline EGE (per 0.5 unit-change) & 0.832 & 3.433 & 0.0012 & & & & \\
\hline $\operatorname{ECV}(\%)$ & 0.595 & 2.987 & 0.0501 & & & & \\
\hline T2-mapping (msec) & 0.082 & 1.414 & 1.0000 & & & & \\
\hline
\end{tabular}

MCP, minmax concave penalty; VRD, ventricular rhythm disturbance; mFDR, marginal false discovery rate; EGE/LGE, early/late gadolinium enhancement; ECV, extracellular volume fraction.

Table V. Results of MCP-logistic regression analysis for differentiating VRD patients with VT from those without VT.

\begin{tabular}{lrrrrrr}
\hline Variables & Estimate & z-value & mFDR & $\begin{array}{c}\text { Average } \\
\text { mFDR }\end{array}$ & $\begin{array}{r}\text { Cross-validated } \\
\mathrm{R}^{2}\end{array}$ & $\begin{array}{c}\text { Signal-to-noise } \\
\text { ratio }\end{array}$ \\
\hline RVEDV/BSA & -0.0105 & -2.351 & 0.327 & 0.578 & $<0.001$ & $<0.001$ \\
Native T1-mapping (per 10 msec) & 0.0005 & 1.761 & 0.830 & & & 0.48 \\
\hline
\end{tabular}

MCP, minmax concave penalty; VT, non-sustained ventricular tachycardia; mFDR, marginal false discovery rate; RVEDV, right ventricular end-diastolic volume; BSA, body surface area.

primary outcome. There was a trend for indexed RVEDV as an independent predictor, but this did not reach statistical significance [odds ratio (OR) 1.07 (95\% CI 1.00-1.16), $\mathrm{P}=0.066]$. Most tissue characterization indices independently predicted the primary outcome with increasing values, with LGE and EGE having the greatest contribution. The presence of LGE namely predicted the primary outcome perfectly and EGE had an OR of 6.35 (95\% CI 2.19-21.62) per 0.5-unit change for the primary outcome in multivariable analysis $(\mathrm{P}<0.001)$. At $\geq 1.3$ cut-off, EGE had a sensitivity and specificity of 88 and $85 \%$, respectively for predicting the primary outcome with $87.1 \%$ accuracy (area under the receiver operator characteristics curve: 0.935). Conversely, only indexed LV/RV EDV/ESV were negative independent predictors of the secondary endpoint. EGE and native T1-mapping trended towards significance as independent predictors of the secondary outcome but did not reach statistical significance [OR $(95 \% \mathrm{CI}): 0.82(0.65-1.03)$, $\mathrm{P}=0.090$ and 1.10 (0.99-1.21), $\mathrm{P}=0.073$, respectively].

A penalized logistic regression analysis employing the MCP penalization method was used for feature selection as described in the methods section. The results for both the primary and secondary outcomes are presented in Tables IV and $\mathrm{V}$, respectively. For the primary outcome, LGE, EGE, ECV and T2-mapping were selected. For the secondary outcome, indexed RVEDV and native T1-mapping were the selected features. Feature reliability can be evaluated using the mFDR of each selected feature. Based on this, for the primary end point the first three features are the least likely to be false discoveries (Table IV). For the secondary endpoint, indexed RVEDV is least likely to be a false discovery although with a relatively high mFDR. As a sensitivity analysis, the MCP penalized regression was re-run for the primary outcome using only the subset with VT vs. controls and no-VT vs. controls separately. However, the results were almost identical to the pooled analysis for the primary outcome. An additional sensitivity analysis was run to compare different types of cardiomyopathies (with at least $n \geq 5$ patients) with regard to baseline characteristics using standard univariable statistics (Table VI). Only the proportion of patients with pathologic T2-mapping values was significantly different between groups. Namely it was present only in patients with myocarditis and DMD/BMD [9 (45\%) and 1 (20\%), respectively] and absent in all other types of cardiomyopathies $(\mathrm{P}=0.013)$.

\section{Discussion}

In a cohort of 50 asymptomatic young athletes presenting for PPE and referred for additional evaluation due to documented VRDs with otherwise normal standard examination with echocardiographic evaluation, CMR identified arrhythmogenic substrates in all participants. In athletes with VRDs other than VT the predominant cardiac condition was recent-onset myocarditis, while the majority of those with VT were diagnosed with ARVC. Tissue characterization indices discriminated between cases and a cohort of 20 healthy control athletes independent of age and sex, as did indexed LVEDV and RVEF. Based on penalized regression analysis, the most valuable indices for discriminating between case-control status were LGE, EGE, ECV and T2-mapping in that order. Only indexed ventricular volumes independently discriminated between athletes with VRD with and without VT. Penalized regression analysis identified indexed RVEDV and native T1-mapping as the most useful indices for VT vs. no-VT discrimination, albeit with a high error margin.

Our findings suggest a multifaceted contribution of CMR in PPE. Next to its ability to assess tissue changes in the 


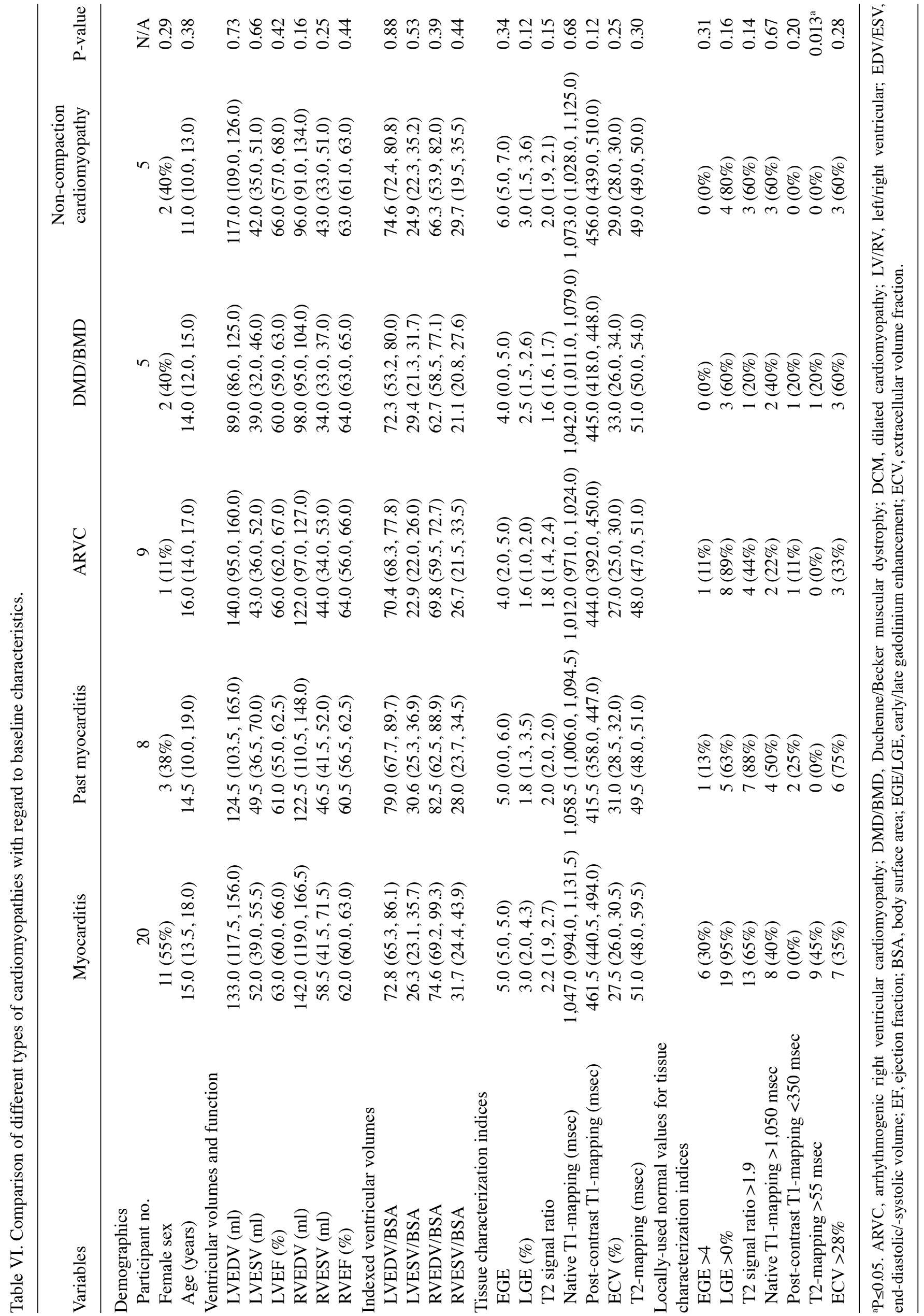


myocardium, CMR also led to the establishment of a new diagnosis in all athletes in this cohort. This is in agreement with previous studies performed by our group and others albeit in adult populations $(28,29)$. Interestingly, although ventricular function did not differ significantly between athletes with VRD and healthy controls, this was not the case for CMR-derived tissue characterization indices, of which, LGE, EGE and ECV had the best discriminatory value. CMR findings in conjunction with clinical assessment allowed the diagnosis of acute and/or chronic cardiac pathology that clarified the observed arrhythmogenicity despite normal echocardiographic findings.

The diagnostic contribution of CMR in the workup of patients with suspected myocarditis/cardiomyopathies is generally well represented in the literature. ARVC in particular may be detected in endurance athletes with VRDs and a normal RV structure, as well as function-wise (30). Additionally, $50 \%$ of patients with non-compaction cardiomyopathy may not be identified using echocardiographic evaluation alone, instead requiring disease demonstration with CMR $(31,32)$. In children with HCM, myocardial fibrosis identified using LGE was associated with adverse events (15) and diffuse ventricular fibrosis identified by T1-mapping predicted non-sustained VT and aborted SCD in adult HCM patients (33). CMR has recently been shown to aid in the workup of patients with seemingly idiopathic VRDs, which by extension can facilitate further diagnostic and therapeutic decision making (34). Furthermore, a normal CMR examination corresponded to a low annual risk of adverse events in a large cohort of patients with suspected myocarditis (29). In patients with thyroid disease CMR identified myocardial inflammation using T2 signal ratio (28). Lastly, in both patients and carriers of DMD, CMR can uniquely reveal myocardial fibrosis as areas of LGE $(35,36)$ or pathologically elevated T1-mapping (37) functioning as arrhythmogenic loci. The more novel CMR indices, namely T1-mapping, T2-mapping and $\mathrm{ECV}$, have also recently been incorporated in the routine CMR evaluation of patients with (suspected) myocarditis or cardiomyopathies (38), signifying their transition from research tools to indices capable of guiding clinical practice.

To our knowledge, this is the only study in the literature presenting a CMR-PPE assessment of young athletes presenting with VRDs and normal echocardiographic evaluation. Our study demonstrates that suspicious ECG findings not corroborated by echocardiography should motivate further assessment using CMR in the context of PPE. In these cases, CMR not only allowed for the establishment of clinical diagnoses with confidence, but also prompted limitation of physical exercise and appropriate diagnostic and therapeutic follow-up according to current practice guidelines. Our findings thus suggest that in cases where echocardiography cannot guide clinical decision making due to equivocal results, CMR can provide an additive, diagnostic value. An additional innovation of this study was the inclusion of penalized regression analyses that allowed the optimal selection of features such that external validity is optimized. To our knowledge this method has not been employed in previous CMR studies except for a single study by our group (39) and a single methodological study using brain MR, which yielded highly accurate feature selection (26).

PPE in adolescents is considered to have an unfavorable cost-benefit trade-off, with one study even suggesting that adding ECG evaluation to PPE at a cost of $\$ 50.000 /$ quality adjusted life year is not cost-effective mainly due to false-positive findings (40). Our study provides for the first time evidence against this claim, as the authors do not mention whether CMR was used in cases of pathologic ECG findings, instead grouping all interventions into a collective 'referral to cardiology'. The study did demonstrate that the addition of ECG led to identification of additional SCD patients, despite not being cost-effective. Our findings, however, suggest that false positive findings might in fact be falsely ruled-out and could be reclassified after further investigation with CMR. Although we did not systematically investigate cost-effectiveness and it is clearly impractical to recommend a CMR examination for all athletes with pathologic ECG findings, our study raises doubts regarding the currently perceived notions around the cost-effectiveness of PPE, which should be reiterated in future studies after the inclusion of CMR.

Our study has some limitations. Only athletes with VRDs were referred to our tertiary center for CMR evaluation, thus introducing a potential selection bias. The predictive capacity of CMR-PPE could not be evaluated due to lack of sufficient clinical follow-up. Additionally, we did not evaluate a uniform population but rather an ensemble of different cardiac pathologies, which may have skewed our results depending on which condition was most prevalent. However, only the proportion of patients with pathologic T2-mapping values differed significantly between the most prevalent types of cardiac pathology. This might have additionally influenced the analysis of the secondary outcome for the same reason, as the VT and non-VT groups had a different prevalence of cardiac pathologies.

To conclude, in this case-control study it was demonstrated for the first time that young athletes presenting with a request for PPE and having VRDs and normal echocardiograms can be optimally discriminated from healthy control athletes using the CMR-derived indices LGE, EGE, ECV and T2-mapping. Furthermore, CMR provided valuable diagnostic utility by allowing for the identification of arrhythmogenic substrates in all cases. This brings current notions regarding the cost-effectiveness of ECG and CMR in PPE into doubt. However, further multicenter studies are needed to assess the cost-effectiveness of this approach, to establish a selection algorithm for CMR-PPE and to evaluate the potential improvements in long-term clinical outcome with the addition of CMR in PPE.

\section{Acknowledgements}

Not applicable.

\section{Funding}

No funding was received.

\section{Availability of data and materials}

The datasets used and/or analyzed during the current study are available from the corresponding author on reasonable request.

\section{Authors' contributions}

GMM conceptualized and designed the study, analyzed and interpreted the data, and was a major contributor in drafting the manuscript. AG, NA, GP, VV, GK, FB and KT, made substan- 
tial contributions to acquisition and interpretation of the data and were involved in drafting the manuscript. CKG and DAS critically revised the manuscript for important intellectual content. SIM supervised the collection and interpretation of data, was involved in drafting the manuscript and critically revised the manuscript for important intellectual content. All authors read and approved the final manuscript.

\section{Ethics approval and consent to participate}

The study was approved by the medical ethics committee of the Aghia Sophia Children's Hospital (protocol no. 27499/29-11-17) and written informed consent was obtained from the parents/legal guardians of the participants.

\section{Patient consent for publication}

The athletes' parents/guardians provided written informed consent for the publication of any associated data.

\section{Competing interests}

The authors are responsible for the choice and presentation of views contained in this article and for opinions expressed therein, which are not necessarily those of UNESCO and do not commit the Organization. DAS is the Editor-in-Chief for the journal, but had no personal involvement in the reviewing process, or any influence in terms of adjudicating on the final decision, for this article. The other authors declare that they have no competing interests.

\section{References}

1. Mirabelli MH, Devine MJ, Singh J and Mendoza M: The Preparticipation Sports Evaluation. Am Fam Physician 92: 371-376, 2015

2. Glover DW and Maron BJ: Profile of preparticipation cardiovascular screening for high school athletes. JAMA 279: 1817-1819, 1998.

3. Toresdahl BG, Rao AL, Harmon KG and Drezner JA: Incidence of sudden cardiac arrest in high school student athletes on school campus. Heart Rhythm 11: 1190-1194, 2014.

4. Fudge J, Harmon KG, Owens DS, Prutkin JM, Salerno JC, Asif IM, Haruta A, Pelto H, Rao AL, Toresdahl BG and Drezner JA: Cardiovascular screening in adolescents and young adults: A prospective study comparing the Pre-participation Physical Evaluation Monograph 4th Edition and ECG. Br J Sports Med 48: 1172-1178, 2014.

5. Gleason CN, Kerkhof DL, Cilia EA, Lanyi MA, Finnoff J, Sugimoto D and Corrado GD: Early screening for cardiovascular abnormalities with preparticipation echocardiography: Feasibility Study. Clin J Sport Med 27: 423-429, 2017.

6. Raukar N, Arciero E, Noyes A, Drezner J and Weiss J: Cardiovascular pre-participation screening in the young athlete: Addressing concerns. Phys Sportsmed 45: 365-369, 2017.

7. Mavrogeni S, Markousis-Mavrogenis G, Koutsogeorgopoulou L and Kolovou G: Cardiovascular magnetic resonance imaging: Clinical implications in the evaluation of connective tissue diseases. J Inflamm Res 10: 55-61, 2017.

8. Nagueh SF, Bierig SM, Budoff MJ, Desai M, Dilsizian V, Eidem B, Goldstein SA, Hung J, Maron MS, Ommen SR, et al; American Society of Echocardiography; American Society of Nuclear Cardiology; Society for Cardiovascular Magnetic Resonance; Society of Cardiovascular Computed Tomography: American Society of Echocardiography clinical recommendations for multimodality cardiovascular imaging of patients with hypertrophic cardiomyopathy: Endorsed by the American Society of Nuclear Cardiology, Society for Cardiovascular Magnetic Resonance, and Society of Cardiovascular Computed Tomography. J Am Soc Echocardiogr 24: 473-498, 2011.
9. Moon JCC, Fisher NG, McKenna WJ and Pennell DJ: Detection of apical hypertrophic cardiomyopathy by cardiovascular magnetic resonance in patients with non-diagnostic echocardiography. Heart 90: 645-649, 2004

10. Mavrogeni SI,Kitas GD,Dimitroulas T,Sfikakis PP,SeoP, Gabriel S, Patel AR, Gargani L, Bombardieri S, Matucci-Cerinic M, et al: Cardiovascular magnetic resonance in rheumatology: Current status and recommendations for use. Int J Cardiol 217: 135-148, 2016.

11. Mavrogeni SI, Sfikakis PP, Markousis-Mavrogenis G, Bournia VK, Poulos G, Koutsogeorgopoulou L, Karabela G, Stavropoulos E, Katsifis G, Boki K, et al: Cardiovascular magnetic resonance imaging pattern in patients with autoimmune rheumatic diseases and ventricular tachycardia with preserved ejection fraction. Int J Cardiol 284: 105-109, 2019.

12. Deal BJ, Miller SM, Scagliotti D, Prechel D, Gallastegui JL and Hariman RJ: Ventricular tachycardia in a young population without overt heart disease. Circulation 73: 1111-1118, 1986.

13. Mavrogeni S, Petrou E, Kolovou G, Theodorakis G and Iliodromitis E: Prediction of ventricular arrhythmias using cardiovascular magnetic resonance. Eur Heart J Cardiovasc Imaging 14: 518-525, 2013.

14. Valente AM, Gauvreau K, Assenza GE, Babu-Narayan SV, Schreier J, Gatzoulis MA, Groenink M, Inuzuka R, Kilner PJ, Koyak Z, et al: Contemporary predictors of death and sustained ventricular tachycardia in patients with repaired tetralogy of Fallot enrolled in the INDICATOR cohort. Heart 100: 247-253, 2014.

15. Spinner JA, Noel CV, Denfield SW, Krishnamurthy R, Jeewa A, Dreyer WJ and Maskatia SA: Association of late gadolinium enhancement and degree of left ventricular hypertrophy assessed on cardiac magnetic resonance imaging with ventricular tachycardia in children with hypertrophic cardiomyopathy. Am J Cardiol 117: 1342-1348, 2016.

16. Friedrich MG, Sechtem U, Schulz-Menger J, Holmvang G, Alakija P, Cooper LT, White JA, Abdel-Aty H, Gutberlet M, Prasad S, et al; International Consensus Group on Cardiovascular Magnetic Resonance in Myocarditis: Cardiovascular magnetic resonance in myocarditis: A JACC White Paper. J Am Coll Cardiol 53: 1475-1487, 2009.

17. Bokma JP, Geva T, Sleeper LA, Babu Narayan SV, Wald R, Hickey K, Jansen K, Wassall R, Lu M, Gatzoulis MA, et al: A propensity score-adjusted analysis of clinical outcomes after pulmonary valve replacement in tetralogy of Fallot. Heart 104: 738-744, 2018.

18. Won S, Davies-Venn C, Liu S and Bluemke DA: Noninvasive imaging of myocardial extracellular matrix for assessment of fibrosis. Curr Opin Cardiol 28: 282-289, 2013.

19. Messroghli DR, Radjenovic A, Kozerke S, Higgins DM, Sivananthan MU and Ridgway JP: Modified Look-Locker inversion recovery (MOLLI) for high-resolution T1 mapping of the heart. Magn Reson Med 52: 141-146, 2004.

20. Etoom Y, Govindapillai S, Hamilton R, Manlhiot C, Yoo SJ, Farhan M, Sarikouch S, Peters B, McCrindle BW and Grosse-Wortmann L: Importance of CMR within the Task Force Criteria for the diagnosis of ARVC in children and adolescents. J Am Coll Cardiol 65: 987-995, 2015.

21. Alhabshan F, Smallhorn JF, Golding F, Musewe N, Freedom RM and Yoo SJ: Extent of myocardial non-compaction: Comparison between MRI and echocardiographic evaluation. Pediatr Radiol 35: 1147-1151, 2005.

22. Mavrogeni SI, Markousis-Mavrogenis G, Papavasiliou A, Papadopoulos G and Kolovou G: Cardiac involvement in duchenne muscular dystrophy and related dystrophinopathies. Methods Mol Biol 1687: 31-42, 2018.

23. Breheny $\mathrm{P}$ and Huang J: Coordinate descent algorithms for nonconvex penalized regression, with applications to biological feature selection. Ann Appl Stat 5: 232-253, 2011.

24. Miller RE and Breheny P: Marginal false discovery rate control for likelihood-based penalized regression models. Biom J 61: 889-901, 2019.

25. Breheny PJ: Marginal false discovery rates for penalized regression models. Biostatistics 20: 299-314, 2019.

26. Liu H, Du G, Zhang L, Lewis MM, Wang X, Yao T, Li R and Huang X: Folded concave penalized learning in identifying multimodal MRI marker for Parkinson's disease. J Neurosci Methods 268: 1-6, 2016.

27. Mehta P, Wang CH, Day AGR, Richardson C, Bukov M, Fisher CK and Schwab DJ: A high-bias, low-variance introduction to Machine Learning for physicists. Phys Rep 810: 1-124, 2019. 
28. Mavrogeni S, Markussis V, Bratis K, Mastorakos G, Sidiropoulou EJ, Papadopoulou E and Kolovou G: Hyperthyroidism induced autoimmune myocarditis. Evaluation by cardiovascular magnetic resonance and endomyocardial biopsy. Int J Cardiol 158: 166-168, 2012.

29. Gräni C, Eichhorn C, Bière L, Murthy VL, Agarwal V, Kaneko K, Cuddy S, Aghayev A, Steigner M, Blankstein R, et al: Prognostic value of cardiac magnetic resonance tissue characterization in risk stratifying patients with suspected myocarditis. J Am Coll Cardiol 70: 1964-1976, 2017.

30. Hedley JS, Al Mheid I, Alikhani Z, Pernetz MA and Kim JH: Arrhythmogenic right ventricular cardiomyopathy in an endurance athlete presenting with ventricular tachycardia and normal right ventricular function. Tex Heart Inst J 44: 290-293, 2017.

31. Diwadkar S, Nallamshetty L, Rojas C, Athienitis A, Declue C, Cox C, Patel A and Chae SH: Echocardiography fails to detect left ventricular noncompaction in a cohort of patients with noncompaction on cardiac magnetic resonance imaging. Clin Cardiol 40: 364-369, 2017.

32. Mavrogeni SI, Markousis-Mavrogenis G, Vartela V, Manolopoulou D, Abate E, Hamadanchi A, Rigopoulos AG, Kolovou G and Noutsias M: The pivotal role of cardiovascular imaging in the identification and risk stratification of non-compaction cardiomyopathy patients. Heart Fail Rev: Nov 30, 2019 (Epub ahead of print).

33. Lu M, Zhao S, Yin G, Jiang S, Zhao T, Chen X, Tian L, Zhang Y, Wei Y, Liu Q, et al: T1 mapping for detection of left ventricular myocardial fibrosis in hypertrophic cardiomyopathy: A preliminary study. Eur J Radiol 82: e225-e231, 2013.

34. Muser D, Santangeli P, Selvanayagam JB and Nucifora G: Role of cardiac magnetic resonance imaging in patients with idiopathic ventricular arrhythmias. Curr Cardiol Rev 15: 12-23, 2019.

35. Mavrogeni S, Bratis K, Papavasiliou A, Skouteli E, Karanasios E, Georgakopoulos D, Kolovou G and Papadopoulos G: CMR detects subclinical cardiomyopathy in mother-carriers of Duchenne and Becker muscular dystrophy. JACC Cardiovasc Imaging 6: 526-528, 2013.
36. Mavrogeni S, Papavasiliou A, Spargias K, Constandoulakis P, Papadopoulos G, Karanasios E, Georgakopoulos D, Kolovou G, Demerouti E, Polymeros S, et al: Myocardial inflammation in Duchenne Muscular Dystrophy as a precipitating factor for heart failure: A prospective study. BMC Neurol 10: 33, 2010.

37. Olivieri LJ, Kellman P, McCarter RJ, Cross RR, Hansen MS and Spurney CF: Native T1 values identify myocardial changes and stratify disease severity in patients with Duchenne muscular dystrophy. J Cardiovasc Magn Reson 18: 72, 2016.

38. Messroghli DR, Moon JC, Ferreira VM, Grosse-Wortmann L, He T, Kellman P, Mascherbauer J, Nezafat R, Salerno M, Schelbert EB, et al: Clinical recommendations for cardiovascular magnetic resonance mapping of T1, T2, T2* and extracellular volume: A consensus statement by the Society for Cardiovascular Magnetic Resonance (SCMR) endorsed by the European Association for Cardiovascular Imaging (EACVI). J Cardiovasc Magn Reson 19: 75, 2017.

39. Markousis-MavrogenisG,MitsikostasDD,Koutsogeorgopoulou L, Dimitroulas T, Katsifis G, Argyriou P, Apostolou D, Velitsista S, Vartela V, Manolopoulou D, et al: Combined brain-heart magnetic resonance imaging in autoimmune rheumatic disease patients with cardiac cymptoms: Hypothesis generating insights from a cross-sectional study. J Clin Med 9: 447, 2020.

40. Schoenbaum M, Denchev P, Vitiello B and Kaltman JR Economic evaluation of strategies to reduce sudden cardiac death in young athletes. Pediatrics 130: e380-e389, 2012.

(i) $\Theta$ This work is licensed under a Creative Commons Attribution-NonCommercial-NoDerivatives 4.0 International (CC BY-NC-ND 4.0) License. 